\title{
Datchball y Colpbol como recursos para promover la inteligencia interpersonal: Experiencia didáctica aplicada con chicas y chicos de Educación Secundaria Datchball and Colpbol as resources to promote interpersonal intelligence: A didactic experience for girls and boys in High School
}

\author{
Cristina Jaquete Pérez, Elena Ramírez Rico \\ Universidad Complutense de Madrid (España)
}

\begin{abstract}
Resumen. El presente trabajo expone una experiencia didáctica diseñada para trabajar la mejora de la inteligencia interpersonal, a través de la impartición de deportes alternativos, dentro del contexto educativo de Educación Secundaria Obligatoria para la asignatura de Educación Física (EF). Por las características que se contemplan en sus reglamentos, el datchball y el colpbol fueron los deportes elegidos. Asimismo, se llevó a cabo un análisis estadístico con la que estudiar la influencia de los beneficios de estos dos deportes en la inteligencia interpersonal del estudiantado. Proceso, que consistió en analizar el desarrollo de las variables cooperación y empatía, íntimamente relacionadas con la inteligencia interpersonal. La muestra fue de 107 estudiantes (60 chicos y 47 chicas) pertenecientes a los cursos de $2^{\circ}$ y $3^{\circ}$ E.S.O. Para la evaluación de estas dos variables se utilizó el cuestionario de cooperación deportiva de Leo, Sánchez-Miguel, Sánchez-Oliva,Amado \& García-Calvo (2011), y el cuestionario de conducta prosocial de Martorell, González \& Calvo (1998). Estos dos cuestionarios fueron cumplimentados por el alumnado tanto antes como después de la experiencia didáctica para poder así analizar si hubo o no alguna variación en las variables. Los resultados que se obtuvieron reflejaron una mejoría tanto en la variable empatía como en la cooperación incondicionada. Está experiencia, por tanto, da indicios que demuestran la mejora de la inteligencia interpersonal entre el estudiantado de ambos sexos durante las clases de Educación Física.
\end{abstract}

Palabras clave: Inteligencia interpersonal, deportes alternativos, cooperación, empatía y Educación Secundaria Obligatoria.

\begin{abstract}
This paper presents a didactic experience designed to improve interpersonal intelligence, through the teaching of novel alternative sports, and tooking place within the educational context of high school in the subject of Physical Education. Due to the characteristics contemplated in their sports regulations, datchball and colpbol were the sports chosen for its impartation. Furthermore, a statistical evaluation was executed to study the influence of the benefits of these two sports on the student's interpersonal intelligence. This process consisted in analyzing the development of the variables cooperation and empathy, intimately related to interpersonal intelligence. The sample was of 107 students ( 60 boys and 47 girls) belonging to the 2nd and 3rd years of the CSE. For the evaluation of these two variables, the sports cooperation questionnaire from Leo, Sánchez-Miguel, Sánchez-Oliva, Amado \& García-Calvo (2011) and the questionnaire of prosocial behavior from Martorell, González \& Calvo (1998) were selected. These two questionnaires were distributed to the students before and after the didactic experience, in order to analyze if there was any variation in the variables. The results obtained showed an improvement in the empathy variable and in the unconditional cooperation. Therefore, this proposal helps to improve the development of interpersonal intelligence among students of both genders during Physical Education.
\end{abstract}

Keywords: Interpersonal Intelligence, alternative novel sports, cooperation, empathy and Compulsory Secondary Education.

\section{Introducción}

Según Gardner (1995) se pueden hablar de ocho tipos de inteligencias: lógico-matemática, lingüística, musical, cinético-corporal, espacial, naturalista, interpersonal e intrapersonal. Además, añade que cualquier persona posee, en mayor o menor medida, cada una de estas inteligencias.

Aunque Gardner habla de ellas de forma separada,

Fecha recepción: 06-01-20. Fecha de aceptación: 20-04-21

Cristina Jaquete Pérez

mjaquete@ucm.es lo cierto es que se pueden encontrar numerosos autores que hablan de manera conjunta sobre la inteligencia interpersonal y la inteligencia intrapersonal, denominándolas inteligencia emocional. La inteligencia emocional se caracteriza por la correcta utilización de nuestras emociones (Weisinger \& Cali, 1999) o la «habilidad para procesar la información emocional que incluye la percepción, la asimilación, la comprensión y la dirección de las emociones» (Mayer \& Cobb, 2000, p. 273).

De la misma manera, el alumnado dentro del entorno escolar puede sufrir un escaso desarrollo de la inteligencia emocional. Ello, conllevaría una serie de consecuencias como pueden ser el deterioro de las relaciones entre el alumnado, la salud psicológica y el ren- 
dimiento académico (Extremera \& Fernández-Berrocal, 2003). Además, podría ser el origen de malas conductas escolares (Aguilar, García-Fernández \& Gil del Pino, 2021), así como una introducción en el consumo de sustancias adictivas (Extremera \& Fernández-Berrocal, 2004). Asimismo, tanto como para la vida cotidiana, como para la laboral, se exigen una seria de competencias necesarias para poder convivir en sociedad, con las que poder ser más productivo y obtener un mayor rendimiento personal y profesional. Cualidades o habilidades que se dan por hecho que se poseen, pero que nadie enseña a detectarlas y, en caso de ser necesario, a moldearlas (Goleman, 2015). Así pues, adquirir el conjunto de valores y actitudes necesarias para un desarrollo interpersonal debe ser uno de los objetivos fundamentales de la educación escolar, con la cual se pueda conseguir el pleno desarrollo del alumnado (Cubas-Martinez et al., 2019).

A pesar de su unificación, lo cierto es que la inteligencia emocional está conformada por una serie de habilidades, claramente diferenciadas por habilidades intrapersonales y habilidades sociales. En base a estas habilidades diferenciadas, autores como FernándezBerrocal \& Pacheco (2002) fueron de los primeros en sugerir el trabajo de las diferentes habilidades de la inteligencia emocional por separado. Y autores como Moreno, Saiz \& Esteban (1998) al trabajar la inteligencia emocional en adolescentes, se centran solo en la inteligencia interpersonal; inteligencia mediante la cual somos capaces de relacionarnos y entendernos con los demás (Molero, Saiz \& Esteban, 1998)».

Según Bar-On \& Parker (2018), en su inventario EQ-I (Bar-On Emotional Quotient Inventory) funda un modelo en el que se recoge la inteligencia emocional. En dicho modelo, Bar-On, indica que dentro del componente interpersonal influye la empatía (habilidad que posee una persona para detectar los sentimientos de los demás), las relaciones interpersonales (habilidad para mantener relaciones sociales con otras personas) y la responsabilidad social (persona cooperante que ayuda a construir dentro de un grupo social). En línea con esta última definición, queda claro que las personas se conectan con otras mediante las relaciones sociales. Por ello, aparece el papel de la cooperación. La cooperación es una ayuda o una colaboración que se da entre dos o más sujetos, y se produce cuando se trabaja en equipo y se tiene una meta común como fin (Rey, 2009).

En base a esto, se procedió a la realización de una experiencia didáctica para la mejora de la inteligencia interpersonal en el alumnado de Educación Secundaria
Obligatoria mediante el trabajo de dos tipos de variables: la empatía y la cooperación, las cuales están íntimamente relacionadas con este tipo de inteligencia.

La siguiente pregunta que se sugiere es cómo trabajar la inteligencia emocional, y más específicamente, cómo desarrollar la inteligencia interpersonal en un centro educativo, donde no hay una asignatura dedicada a ello. Aunque parece que el interés por tratar de incorporar una enseñanza basada en las Inteligencias Múltiples está en auge (Constantino \& Navia, 2021). la realidad es que en la actualidad la inteligencia interpersonal aún no está incorporada dentro del modelo curricular educativo español. Ante esta situación, el docente se ve obligado a encontrar una ruta alternativa para tratar de incorporarla.

Puesto que son muchos los autores que señalan la existencia de una relación entre el ejercicio físico y las emociones (Bermudez \& Saenz-López, 2019; Thayer, 2003), el propósito de este trabajo es incorporar el trabajo específico de las emociones, y más concretamente de la inteligencia interpersonal, en el desarrollo de la asignatura de EF. Se pueden encontrar estudios (Roche, 2004; Ros, Moya-Faz \& Garcés de los Fayos, 2013) que se basan en deportes tradicionales con el fin de desarrollar la inteligencia emocional o la inteligencia interpersonal, pero no se han encontrado estudios realizados con deportes alternativos. Es por ello, que partiendo de la efectividad que se recoge sobre estos deportes en diferentes estudios, entre otras cuestiones por su carácter novedoso y su reglamentación, aumentando la motivación, implicación y disfrute hacia la práctica de actividad física, (Escamilla, Alguacil \& González-Serrano, 2020; Lizandra, \& Peiró-Velert, 2019), esta experiencia didáctica se ha basado en utilizar los deportes alternativos como vía de desarrollo de la inteligencia interpersonal.

Así bien, el objetivo pretendido en este trabajo es incorporar una experiencia didáctica diseñada para trabajar la mejora de la inteligencia interpersonal en las clases de EF de un centro educativo, a través de la introducción de dos deportes alternativos: datchball y colpbol.

\section{Metodología}

\section{Contexto del centro}

El contexto de este estudio lo conforman las clases de la asignatura de EF en un centro escolar de la Comunidad de Madrid, en la que el alumnado inscrito proviene de familia con estudios universitarios y con un nivel 
socio - económico alto. Se trata de un centro sin problemáticas reseñables de contexto ni de sujetos. Cabe añadir, que se rige por un modelo de enseñanza tradicional, donde los deportes practicados en las clases de EF son baloncesto, balonmano, voleibol y hockey.

\section{Participantes}

El alumnado participante son estudiantes procedentes de los cursos de $2^{\circ}$ y 3 E.S.O. Ambos cursos fueron seleccionados por las posibilidades del momento para la impartición de la propuesta educativa y como estudio de caso. El estudio lo conforman un total de 107 estudiantes, entre los que se encontraban 60 chicos y 47 chicas, de edades comprendidas entre los 13 y los 14 años.

\section{Instrumentos}

Debido a las características que se contemplan en sus respectivos reglamentos, el datchball y el colpbol son los deportes alternativos elegidos para desarrollar los contenidos con los que cumplir el principal objetivo de incorporar una experiencia didáctica diseñada para trabajar la mejora de la inteligencia interpersonal en las clases de EF de un centro educativo. Asimismo, se procedió al análisis sobre cuáles podrían ser los instrumentos que mejor se adaptaran a las exigencias del desarrollo de la inteligencia interpersonal a través del trabajo de las variables cooperación y empatía.

Se usó el cuestionario de cooperación deportiva sobre las relaciones interpersonales entre los jugadores de Leo, Sánchez-Miguel, Sánchez-Oliva, Amado \& García-Calvo (2011), utilizando los factores de cooperación disposicional condicionada, cooperación disposicional incondicionada y cooperación situacional con los compañeros. Puesto que se trata de un cuestionario utilizado en un contexto de entrenamiento deportivo, se adaptó el vocabulario específico, quitando conceptos usados en el ámbito de entrenamiento deportivo por un léxico adaptado al contexto educativo, cómo «Mi cooperación con mis compañeros y el profesor, durante las clases de Educación Física, depende de la colaboración que ellos me dan a mí».

También se utilizó el cuestionario de Martorell, González \& Calvo (1998), encargado de medir las conductas prosociales, entre las que aparecía la variable empatía, y se procedió a la elección de los ítems encargados de medir esta variable. Estos cuestionarios fueron pasados antes de la realización de la experiencia didáctica y posteriormente, para poder así valorar el grado de mejora de las variables de estudio.

\section{Planteamiento didáctico-experiencia}

En base al programa educativo español, los deportes colpbol y datchball pueden ser tratados como deportes de cooperación-oposición, puesto que como equipo se deben trabajar conjuntamente para alcanzar una meta común y es necesario jugar contra un equipo rival. Si además se trabaja en pequeños grupos, se podrán conseguir habilidades interpersonales con las que gestionar, planificar, resolver problemas, tomar decisiones, obtener capacidades de liderazgo o asumir responsabilidades (Balcazar, 2020).

El colpbol, a pesar de que su origen data del año 1997, es un deporte innovador en muchas escuelas. Destaca por tener como principal norma un único golpeo, sin poder retener o agarrar el balón. Esto lo convierte en un deporte muy dinámico, que fomenta la participación de todo el alumnado y reduce las diferencias individuales, ya que exige una colaboración colectiva para lograr el objetivo final: el gol. Se trata de un deporte parecido al fútbol, pero con menor exigencia técnica, que sigue unas líneas lúdicas y socializadoras que fomentan las relaciones interpersonales según su creador Bendicho (2014). Un estudio en Educación Primaria desempeñado por Hernández, Martínez \& Carrión (2019) verifica como el colpbol es un deporte motivante, participativo, y que promueve conductas de empatía y solidaridad.

El datchball es otro deporte emergente de cancha dividida. Es una adaptación del balón prisionero, cuyo objetivo es intentar eliminar a los jugadores del campo contrario golpeándolos con varias pelotas. Se trata de un deporte muy dinámico y participativo. Sus principales normas son reconocer cuándo estás eliminado y animar al equipo cuando esto sucede; por lo que también trabaja valores como el compañerismo, la integración, el respeto y la aceptación de la derrota (Asociación Internacional de Datchball, 2018). Una intervención realizada con estudiantes de Educación Secundaria demuestra cómo a través del datchball se consigue desarrollar emociones positivas (Hamadeh \& Ramírez, 2017). Teniendo en cuenta que la empatía ha sido vinculada por varios autores como un posible medio para el desarrollo psicológico, un incremento emocional positivo supondría una mejora en el desarrollo de la empatía del alumnado (Eisenberg, Zhou, Spinrad, Valiente, Fabes \& Liew, 2005).

La experiencia didáctica, que aquí se presenta, consistió en seis sesiones de unos 45 minutos cada una de ellas (tres sesiones dedicadas al deporte datchball y tres sesiones aplicadas al deporte colpbol) impartidas du- 
$\left(10^{\prime}\right) \quad$ Mano magnética: El alumnado se desplaza por el espacio, y a la señal de mano magnética y un número se agrupa en función de ese número.

Parte principal Cazadores y Liebres: Unas pocas personas harán de cazadores con el objetivo de pillar al resto del alumnado que serán las liebres. Los cazadores, identificados con ayuda de un peto

(30) tendrán que eliminar a las liebres lanzándoles un balón de gomaespuma. Cuando los cazadores tengan el balón en la mano, solo podrán dar tres pasos. El lanzamiento solo será válido si se lanza directamente o con un bote, y a continuación, después de dar a la liebre, el balón vuelve a tocar el suelo. Los balones que den en la pared y luego a una liebre de rebote no serán válidos (reglas de datchball). Si una liebre es dada, se tendrán que sentar en el suelo y podrá volver a incorporarse al juego si otras dos liebres, que no estén sentadas por eliminación, salvan al estudiante agarrándole cada uno de una mano para que se levante.

Los cazadores dispondrán de tres minutos para intentar eliminar a todas las liebres. Se cambiarán los roles cada dicho tiempo.

Invasión de pelotas: Divididos en dos equipos con una cuerda central divisoria colocada a varios cm del suelo. Un equipo en cada lado del campo. Se colocarán varios balones debajo de esta cuerda, y una cuerda por campo. El alumnado estará colocado detrás de esta última cuerda, la cual se encuentra a cierta distancia de la central. Esta cuerda será la que les indicará desde donde se puede lanzar. Se repartirán pelotas de gomaespuma y el estudiantado tendrá el objetivo de lanzar para intentar dar a las pelotas colocadas en centro, intentando mandar las pelotas al campo contrario. Se les dará un tiempo de tres minutos, al parar el crono, el equipo que menos pelotas tenga en su campo será el equipo ganador. El alumnado solo podrá invadir la zona que hay entre la cuerda central y su cuerda para coger las pelotas de gomaespuma. Una vez cogidas volverán a su posición detrás de la cuerda. No podrán golpear las pelotas colocadas en el centro con ninguna parte del cuerpo. No habrá una pelota de gomaespuma por persona, tendrán que ir repartiéndoselas. Se repetirá tres veces.

-Todos para una!: Se agrupará a todo el estudiantado en un gran círculo, y uno/a detrás de otro/a en sentido a las agujas del reloj. Deberán agarrarse con las manos a los hombros del compañero/a que está delante, flexionar rodillas para intentar sentarse sobre las rodillas del compañero/a que está detrás. El alumnado tendrá que aguantar en esta posición durante 3". Variante uno: colocados en esta posición dar 3 palmadas a la vez. Variante dos: colocados en esta misma posición, andar hacia atrás como los cangrejos hasta completar una vuelta en Variante uno: colocados en esta posicion dar 3 palmadas a la vez. Variante dos: colocados en esta misma posicion, andar hacia atrás como los cangrejos hasta completar una vuelta en

Vuelta a la calma Reflexión sobre la clase por parte del alumno. Preguntas del profesorado al alumnado: ¿Os ha gustado la clase? ¿Os ha parecido divertida? En el primer ejercicio, cuando hacíais el

(5') rol de liebre ¿intentabais salvar a los que podías o solo a vuestros amigos/as? ¿con quién solíais poneros de acuerdo para salvar a otra liebre? En el segundo ejercicio, ¿intentabais lanzar siempre o habéis pasado la pelota de gomaespuma a otro compañero/a que no había lanzado? En el tercer ejercicio, ¿̇os molestaba que algún compañero/a no se colocase en la posición requerida?

\begin{tabular}{clc} 
Sesión dos & Materiales & Bancos suecos, cuerdas, pelotas de voleibol y gomaespuma, petos y cronometro. \\
\hline Objetivos & Desarrollar juegos que favorezcan la cooperación-oposición; así como las habilidades motrices básicas y específicas. Mejorar la inteligencia interpersonal.
\end{tabular}

Objetivos Desarrollar juegos que favorezcan la cooperación-oposición; así como las habilidades motrices básicas y específicas. Mejorar la inteligencia interpersonal.

$\begin{array}{cl}\text { (5') } & \text { 2.Bull dog. Varios estudiantes se colocarán en mitad del espacio y deberán pillar al resto de compañeros/as que irán pasando de lado a lado del campo; mientras que el alumnado }\end{array}$ encargado de pillar solo podrá desplazarse transversalmente.

Parte principal Feria de puntería: Divididos en dos equipos, colocado cada uno en una mitad del gimnasio. Cada equipo se subdividirá en dos grupos: tiradores (peto rojo) y recogepelotas (peto azul).

(30') En cada una de las mitades, se colocarán tres bancos suecos a distintas distancias con cuatro conos colocados encima de cada uno de ellos. Los tiradores se colocarán detrás de una cuerda, desde la cual tienen que lanzar pelotas de gomaespuma e intentar tirar los conos que hay colocados. Solo las personas que estén en el primer lugar de la fila tendrán balón. Las personas recogepelotas, estarán colocadas en un lateral del circuito en una fila, tendrán que ayudar a los lanzadores e ir a por la pelota lanzada y dársela al siguiente lanzador de la fila que este sin pelota. Derribar un cono que este colocado en el banco sueco más cercano a la zona del tirador valdrá un punto, dos puntos los conos del banco sueco que se encuentra en el medio y tres pelota. Derribar un cono que este colocado en
puntos los conos del banco sueco más alejado.

puntos los conos del banco sueco más alejado.
Se dará un tiempo de dos minutos y 30 segundos por cada rol. El equipo que haya hecho más puntos es el equipo ganador.

iA barrer la sala!: mismos equipos de antes. Cada equipo deberá recoger los conos y apilarlos en una esquina del gimnasio y colocar los tres bancos suecos seguidos en una línea recta en la mitad de su campo. Se les pondrá como condición que solo se puede llevar un objeto por persona y un banco cada dos personas. El equipo que antes lo haga será el ganador con un punto. Derribando los bolos: Mismos equipos. El alumnado de peto rojo se pasa al campo contrario, colocándose encima de los bancos suecos y juega contra el alumnado de peto azul del otro equipo y viceversa con el otro equipo. El estudiantado que no esté encima del banco deberá lanzar un balón de gomaespuma para intentar dar a una de las personas colocadas en el banco, si le dan, está se sentará y valdrá un punto para el equipo contrario. Además, determinados estudiantes tendrán una pelota de voleibol en sus manos para usar como escudo de defensa, de manera que si la pelota de gomaespuma toca este escudo sumará un punto para el equipo defensor. Otros estudiantes no tendrán pelota como escudo y podrán hacer aire. Si hacen aire el manera que si la pelota de gomaespuma toca este escudo sumará un punto para el equipo defensor. Otros estudiantes no tend
atacante será eliminado y tendrá que sentarse y sumará 1 punto para el equipo defensor. A continuación, se hará cambio de rol.

Vuelta a la calma Atrápame si puedes: mismos equipos. Cada equipo colocado en su campo y con sus petos del grupo al que pertenecen. Tendrán que estar colocados tocando la pared. Se colocarán tres

$\left(10^{\prime}\right)$ pelotas de voleibol en medio del campo sobre las cuerdas divisorias. El profesorado mostrará un peto y gritará datchball, tras esta señal el estudiantado que lleve puesto el mismo color de peto que muestra el profesorado será el alumnado que tendrá que salir a correr a intentar coger una de las pelotas que están en el medio. Hacerse con una pelota valdrá un punto. El equipo que consiga más pelotas es el ganador.

Reflexión sobre la clase por parte del alumnado.

\begin{tabular}{lll} 
Sesión tres & Materiales & Bancos suecos, cuerdas, pelotas de gomaespuma, aros y petos \\
\hline Objetivos & A prender a jugar a datchball y favorecer asíla empatía entre ellos. Mejorar la inteligencia interpersonal.
\end{tabular}

Objetivos Aprender a jugar a datchball y favorecer así la empatía entre ellos. Mejorar la inteligencia interpersonal.

Calentamiento Movilidad articula

(10') Tres en raya: Divididos en cuatro equipos, cada uno con un peto de color diferente, y con ayuda de los aros colocaremos en medio del gimnasio dos tableros de tres en raya. Dos equipos por tablero. Las fichas serán tres petos por equipo del mismo color del peto del equipo. Cada equipo se colocará en fila en uno de los extremos del gimnasio y saldrá corriendo con una ficha que deberá colocar en el tablero, después volverá a la fila y podrá salir la siguiente persona de la fila para colocar su ficha. Los equipos que antes hagan tres en raya ganan y se enfrentarán las personas ganadoras de cada partida.

Parte principal Datchball: Una vez aclaradas todas las normas, el alumnado juega a este deporte alternativo.

$\left(30^{\prime}\right)$

Vuelta a la calma Reflexión sobre la clase por parte de los alumnos.

(5)

\begin{tabular}{clc} 
Sesión cuatro & Materiales & Globos, petos, cronómetro. \\
\hline Objetivos & Aprender los distintos golpeos de colpbol. Favorecer conductas ligadas a la cooperación incondicionada. Mejor la intelgen
\end{tabular}

Objetivos Aprender los distintos golpeos de colpbol. Favorecer conductas ligadas a la cooperación incondicionada. Mejorar la inteligencia interpersonal.

Calentamiento Movilidad articular.

(10') En marcha: En dos equipos, un equipo deberá colocar una fila de conos apilados desde un extremo del campo hasta el otro, solo podrán coger un cono por trayecto, ni podrán lanzarlos. Mientras, el otro equipo correrá dando vueltas al campo hasta que el otro equipo termine su tarea. El equipo que dé más vueltas al campo gana.

Parte principal Punch me: Colocados por parejas, harán carreras de relevos golpeando un globo ida y vuelta según las indicaciones del profesorado, con el objetivo de que aprendan los distintos golpeos

(30') que hay en colpbol.

Globo caliente: en dos equipos, cada uno colocado en una mitad de campo y separados por una línea, el estudiantado tendrá que pasarse los globos sin que caigan al suelo. Se irá dejando un tiempo determinado y cuando se pare el cronómetro ganará el equipo que menos globos tenga en su campo.

Cazando al vuelo: dos equipos distinguidos con ayuda de petos. Cada persona del equipo se colocará dentro de un espacio del que no podrá salir, y deberá lanzar un globo al aire e ir golpeándolo de distintas formas intentando que no caiga al suelo. Mientras, que las personas del otro equipo deberán molestarles sutilmente para intentar que los globos caigan al suelo.

Vuelta a la calma Pinchar los globos. Un equipo hará un círculo y dentro de él, colocará todos los globos que tendrá que proteger para que las personas del equipo contrario no se los exploten.

(5') Estiramientos de todos los músculosimplicados.

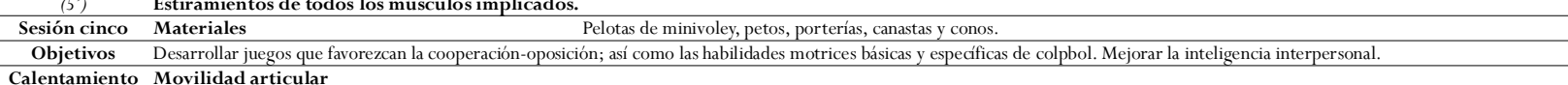

Calentamiento Movilidad articular

$\left(10^{\prime}\right) \quad$ Carrera cooperativa: Colocados en fila y en quipos de tres, el estudiantado deberá desplazarse de un cono a otro. Al comenzar solo saldrá la primera persona de la fila y a su regreso deberá agarrar de la mano a la siguiente persona colocada en la fila. Así sucesivamente hasta realizar el recorrido en una cadena formada por todos los/as integrantes del equipo. El equipo que antes lo complete será el ganador.

Parte principal Cesto partido a dos toques: En un campo de baloncesto, un equipo jugará un partido de colpbol a dos toques por persona y marcarán gol si la pelota toca el tablero de la canasta.

(30')
Partido a dos toques: En otro campo de baloncesto, un segundo equipo jugará un partido de colpbol a dos toques por persona y marcarán gol si la pelota pasa una portería delimitada Rotación de las por dos conos

siguientes actividades. Frontón: El tercer equipo jugará un frontón a dos toques en una pared. Golpeando el balón con la palma de la mano y permitiendo un bote.

Vuelta a la calma Masaje por parejas con el balón de minivoley.

$\left(5^{\prime}\right)$

Sesión seis Materiales Petos, porterías y dos pelotas de colpbol.

Objetivos Aprender a jugar a colpbol y favorecer así la cooperación entre ellos. Mejorar la inteligencia interpersonal.

Calentamiento Movilidad articular

$\left(10^{\prime}\right) \quad$ Come cocos: El estudiantado se moverá por las líneas de campo de colpbol y a continuación se dará salida a cinco come cocos que serán las personas encargadas de ir pillando al resto, siempre pisando las líneas de campo. No se podrá adelantar. Una vez pillados/as se sentarán hasta que pillen al resto de compañeros y compañeras.

Parte principal Colpbol: Una vez aclaradas todas las normas, debido al gran número de estudiantes, el alumnado jugará dos partidos de colpbol En un campo se enfrentarán dos equipos y en otro campo

$\left(30^{\prime}\right) \quad$ los otros dos. Los equipos ganadores de ambos partidos se enfrentarán entre sí hasta que haya un ganador final. También se disputará el partido por el tercer y cuarto puesto.

Vuelta a la calma Reflexión sobre la clase por parte del alumno.

(5') Preguntas del profesorado al alumnado:

-2 Os ha gustado la clase?, ¿Os ha parecido divertida?

¿¿Cómo realizáis los pases para que no os quiten la pelota?, ¿Cómo os debéis poner y qué debéis realizar para cortar el pase?, ¿Notáis más dificultad a la hora de defender?, ¿Y las personas atacantes?, ¿Por qué? 
rante un período de seis semanas.

A continuación, se incluyen las sesiones realizadas en esta experiencia didáctica (Tabla 1).

\section{Análisis de datos}

Para los dos instrumentos, el tratamiento de los datos obtenidos se realizó mediante el Programa Estadístico SPSS v. 23. Las técnicas empleadas fueron aquellas de carácter no paramétrico, dado que la variable cooperación y la variable empatía no siguieron una distribución normal. Se llevó a cabo la prueba de U de MannWhitney para dos muestras independientes para analizar la variable cooperación y empatía en el alumnado por curso y sexo antes y después de la intervención. Para analizar el conjunto de los resultados obtenidos, tanto antes como después de la intervención se realizó la prueba de Wilcoxon de los rangos de signos para dos muestras relacionadas, para así poder analizar y comparar las variables empatía pre y post y cooperación pre y post. Además, se utilizó la Correlación de Spearman para analizar y comparar si hubo alguna relación entre las variables empatía y cooperación, tanto antes como después de la intervención.

Por último, el análisis de fiabilidad de la muestra fue $>$.7 lo cual demuestra una buena consistencia según los valores de referencia (.7-.9) (George \& Mallery, 1995). Hallado el valor de Alfa de Cronbach para el conjunto de ítems previos y de después de la intervención, se obtiene un valor de fiabilidad de .92 .

\section{Resultados más relevantes}

En el análisis de la cooperación y de la empatía por sexo, la U de Mann-Whitney confirmó que no existían diferencias significativas ( $p>$.05) en las variables cooperación y empatía antes y después de la intervención. Salvo el caso de la empatía previa al estudio, cuya p es

Tabla 2

Prueba U de Mann-Whitney para dos muestras independientes en función del sexo

\begin{tabular}{|c|c|c|c|c|c|c|c|c|}
\hline \multicolumn{2}{|c|}{ Entre las variables } & Sexo & $\mathrm{N}$ & Media & $\begin{array}{l}\text { Desviación } \\
\text { típica }\end{array}$ & $\begin{array}{l}\text { Rango } \\
\text { promedio }\end{array}$ & Z & Sig. \\
\hline \multirow{4}{*}{$\begin{array}{l}\text { Cooperación } \\
\text { condicionada }\end{array}$} & \multirow{2}{*}{ PRE } & Chicos & 60 & 3.49 & .87 & 56.81 & \multirow{2}{*}{-1.06} & \multirow{2}{*}{.28} \\
\hline & & Chicas & 47 & 3.43 & .69 & 50.41 & & \\
\hline & \multirow{2}{*}{ POST } & Chicos & 60 & 3.54 & .78 & 56.74 & \multirow{2}{*}{-1.03} & \multirow{2}{*}{.30} \\
\hline & & Chicas & 47 & 3.42 & .68 & 50.50 & & \\
\hline \multirow{4}{*}{$\begin{array}{l}\text { Cooperación } \\
\text { incondicionada }\end{array}$} & \multirow{2}{*}{ PRE } & Chicos & 60 & 3.74 & .74 & 52.03 & \multirow{2}{*}{-.75} & \multirow{2}{*}{.45} \\
\hline & & Chicas & 47 & 3.88 & .55 & 56.52 & & \\
\hline & \multirow{2}{*}{ POST } & Chicos & 60 & 3.99 & .58 & 55.60 & \multirow{2}{*}{-.60} & \multirow[t]{2}{*}{.54} \\
\hline & & Chicas & 47 & 3.86 & .76 & 51.96 & & \\
\hline \multirow{4}{*}{$\begin{array}{c}\text { Cooperación } \\
\text { Situacional de } \\
\text { los compañeros }\end{array}$} & \multirow{2}{*}{ PRE } & Chicos & 60 & 3.77 & .98 & 49.52 & \multirow{2}{*}{-1.73} & \multirow{2}{*}{.08} \\
\hline & & Chicas & 47 & 4.11 & .74 & 59.72 & & \\
\hline & \multirow{2}{*}{ POST } & Chicos & 60 & 3.94 & .93 & 50.83 & \multirow{2}{*}{-1.21} & \multirow{2}{*}{.22} \\
\hline & & Chicas & 47 & 4.14 & 88 & 58.04 & & \\
\hline \multirow{4}{*}{$\begin{array}{l}\text { Cooperación } \\
\text { Total }\end{array}$} & \multirow{2}{*}{ PRE } & Chicos & 60 & 3.65 & .64 & 52.88 & \multirow[b]{2}{*}{-.42} & \multirow[b]{2}{*}{.67} \\
\hline & & Chicas & 47 & 3.74 & .46 & 55.44 & & \\
\hline & \multirow{2}{*}{ POST } & Chicos & 60 & 3.80 & .51 & 55.21 & \multirow{2}{*}{-.45} & \multirow{2}{*}{.64} \\
\hline & & Chicas & 47 & 3.74 & .59 & 52.46 & & \\
\hline \multirow{4}{*}{ Empatía } & \multirow{2}{*}{ PRE } & Chicos & 60 & 2.75 & .46 & 48.22 & \multirow{2}{*}{-2.18} & \multirow{2}{*}{.02} \\
\hline & & Chicas & 47 & 2.90 & .48 & 61.38 & & \\
\hline & \multirow{2}{*}{ POST } & Chicos & 60 & 2.88 & .50 & 51.76 & & 9 \\
\hline & & Chicas & 47 & 2.95 & .48 & 56.86 & -.84 & .39 \\
\hline
\end{tabular}

menor de .05 (tabla 2). En este caso, sí que existen diferencias entre sexos. Teniendo en cuenta la media, la desviación típica y el rango promedio en esta variable, las chicas tenían una mayor empatía que los chicos previamente a la intervención.

En el análisis de las variables por curso, la U de MannWhitney confirmó que no existían diferencias significativas $(\mathrm{p}>.05)$ en las variables de cooperación. Sin embargo, sí habría diferencias significativas en la variable empatía. Estas diferencias se especifican en que tanto antes como después de la intervención, el grado de empatía es mayor en $2^{\circ} \mathrm{ESO}$ ya que la media y el rango promedio (tabla 3) es más elevado en este curso.

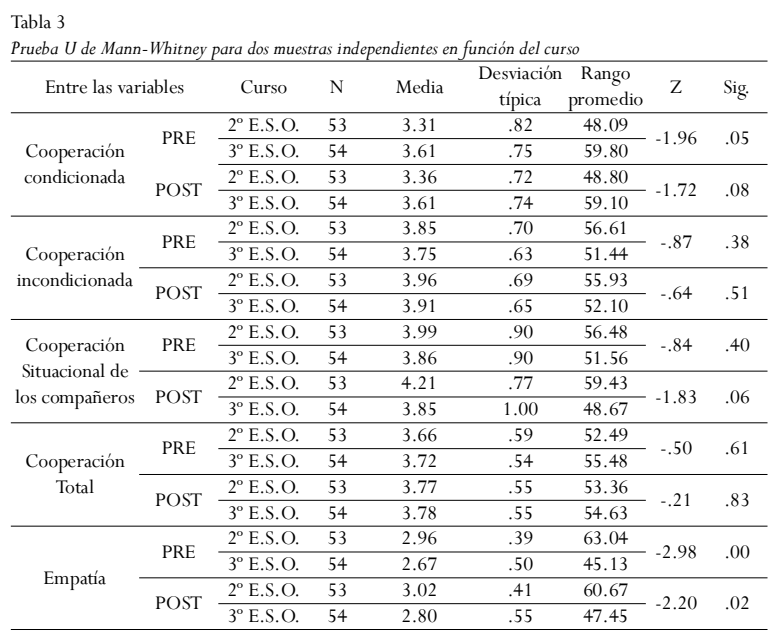

$\mathrm{Al}$ analizar las variables por sexo (tabla 4) se aprecia que existen diferencias significativas en el antes y el después. En el caso de los chicos, en la cooperación incondicionada, la cooperación total y la empatía sí existen diferencias estadísticamente significativas, puesto que el $\mathrm{p}<.05$. Teniendo en cuenta la media y la desviación estándar de cada una de ellas, se puede afirmar que los chicos experimentan una mejora en estas tres variables tras haberse llevado a cabo la intervención con los Tabla 4

\begin{tabular}{|c|c|c|c|c|c|c|}
\hline \multicolumn{7}{|c|}{ Chicos } \\
\hline \multicolumn{2}{|l|}{ Entre las variables } & \multirow{3}{*}{$\begin{array}{l} \\
60 \\
60\end{array}$} & \multirow{2}{*}{$\begin{array}{c}\text { Media } \\
3.49\end{array}$} & \multirow{2}{*}{$\begin{array}{c}\text { Desviación típica } \\
.87 \\
\end{array}$} & \multirow{3}{*}{$\begin{array}{c}\mathrm{Z} \\
-.41\end{array}$} & \multirow{3}{*}{$\begin{array}{l}\text { Sig. } \\
.67\end{array}$} \\
\hline \multirow{2}{*}{ Cooperación condicionada - } & PRE & & & & & \\
\hline & POST & & 3.54 & .78 & & \\
\hline Cooperación & PRE & 60 & 3.74 & .74 & \multirow{2}{*}{-3.08} & \multirow{2}{*}{.00} \\
\hline incondicionada & POST & 60 & 3.99 & .58 & & \\
\hline \multirow{2}{*}{$\begin{array}{c}\text { Cooperación Situacional de } \\
\text { los compañeros }\end{array}$} & PRE & 60 & 3.77 & 98 & \multirow{2}{*}{-1.20} & \multirow{2}{*}{.22} \\
\hline & POST & 60 & 3.94 & .93 & & \\
\hline \multirow{2}{*}{ Cooperación Total } & PRE & 60 & 3.65 & .64 & \multirow{2}{*}{-2.28} & \multirow{2}{*}{.02} \\
\hline & POST & 60 & 3.80 & .51 & & \\
\hline \multirow{2}{*}{ Empatía } & PRE & 60 & 2.75 & .46 & \multirow{2}{*}{-2.74} & \multirow{2}{*}{.00} \\
\hline & POST & 60 & 2.88 & .50 & & \\
\hline \multicolumn{7}{|c|}{ Chicas } \\
\hline \multicolumn{2}{|l|}{ Entre las variables } & $\mathrm{N}$ & Media & Desviación típica & $\mathrm{Z}$ & Sig. \\
\hline \multirow{2}{*}{ Cooperación condicionada } & PRE & 47 & 3.43 & .69 & \multirow{2}{*}{-.97} & \multirow{2}{*}{.33} \\
\hline & POST & 47 & 3.42 & .68 & & \\
\hline Cooperación & PRE & 47 & 3.88 & .55 & \multirow{2}{*}{-.41} & \multirow{2}{*}{.67} \\
\hline incondicionada & POST & 47 & 3.86 & .76 & & \\
\hline \multirow{2}{*}{$\begin{array}{c}\text { Cooperación Situacional de } \\
\text { los compañeros } \\
\end{array}$} & PRE & 47 & 4.11 & .74 & \multirow{2}{*}{-.44} & \multirow{2}{*}{.65} \\
\hline & POST & 47 & 4.14 & .88 & & \\
\hline \multirow{2}{*}{ Cooperación Total } & PRE & 47 & 3.74 & .46 & \multirow[b]{2}{*}{-.59} & \multirow{2}{*}{.55} \\
\hline & POST & 47 & 3.74 & .59 & & \\
\hline \multirow{2}{*}{ Empatía } & PRE & 47 & 2.90 & .48 & \multirow{2}{*}{-.72} & 46 \\
\hline & POST & 47 & 2.95 & .48 & & 1.0 \\
\hline
\end{tabular}


deportes alternativos. Con respecto a las chicas, estadísticamente no se podría afirmar que exista una variación significativa en estas variables por ser el valor $\mathrm{p}>.05$.

En cuanto al análisis en función del curso no se podría establecer ninguna evidencia estadísticamente significativa en el curso de $2^{\circ}$ ESO. Sin embargo, en $3^{\circ}$ ESO sí que existen diferencias significativas en las variables cooperación incondicionada y empatía al ser p $<.05$ (tabla 5). En este caso habría que elegir la hipótesis alternativa. Teniendo en cuenta la media y la desviación típica en estos dos casos, tanto la cooperación incondicionada como la empatía mejoran, al ser mayor la media y la desviación típica después de la intervención.

Tabla 5

Prueba deWilcoxon para dos muestras independientes en función del curso ( $\left.3^{\circ} \mathrm{ESO}\right)$

\begin{tabular}{|c|c|c|c|c|c|c|}
\hline \multicolumn{2}{|c|}{ Entre las variables } & \multirow{3}{*}{$\begin{array}{l}\mathrm{N} \\
54 \\
54\end{array}$} & \multirow{2}{*}{$\begin{array}{c}\text { Media } \\
3.61\end{array}$} & \multirow{3}{*}{$\begin{array}{c}\text { Desviación típica } \\
.75 \\
.74\end{array}$} & \multirow{3}{*}{$\begin{array}{c}\mathrm{Z} \\
-.55\end{array}$} & \multirow{3}{*}{$\begin{array}{l}\text { Sig. } \\
.57\end{array}$} \\
\hline Cooperación & PRE & & & & & \\
\hline condicionada & POST & & 3.61 & & & \\
\hline \multirow{2}{*}{$\begin{array}{c}\text { Cooperación } \\
\text { incondicionada }\end{array}$} & PRE & 54 & 3.75 & .63 & \multirow{2}{*}{-2.58} & \multirow{2}{*}{.01} \\
\hline & POST & 54 & 3.91 & .65 & & \\
\hline \multirow{2}{*}{$\begin{array}{l}\text { Cooperación Situacional } \\
\text { de los compañeros }\end{array}$} & PRE & 54 & 3.86 & .90 & \multirow{2}{*}{-.07} & \multirow{2}{*}{.93} \\
\hline & POST & 54 & 3.85 & 1.00 & & \\
\hline \multirow{2}{*}{ Cooperación Total } & PRE & 54 & 3.72 & .54 & \multirow{2}{*}{-1.06} & \multirow{2}{*}{.28} \\
\hline & POST & 54 & 3.78 & .55 & & \\
\hline \multirow{2}{*}{ Empatía } & PRE & 54 & 2.67 & .50 & \multirow{2}{*}{-2.20} & \multirow{2}{*}{.02} \\
\hline & POST & 54 & 2.80 & .550 & & \\
\hline
\end{tabular}

Con respecto a la cooperación incondicionada y la empatía previa a la intervención, dado que el p-valor es menor a .05 , rechazamos la hipótesis nula. Por consiguiente, existe suficiente evidencia estadística para afirmar que la percepción sobre la cooperación incondicionada pre y la empatía pre está relacionada significativamente. Por otro lado, el coeficiente de correlación de Spearman $=.44$, indica una relación positiva de nivel moderado, a mejor cooperación incondicionada previa, mejor empatía previa. El p-valor de la cooperación situacional del alumnado y la cooperación total vuelve a ser menor de .05 (tabla 6). Por lo tanto, volvemos a rechazar la hipótesis nula, pudiendo afirmar estadísticamente que la empatía está relacionada con las dos variables anteriormente citadas. En el caso de la cooperación situacional del alumnado, la correlación de Spearman es .39 y en la cooperación total .32, habiendo una relación baja-moderada entre dichas variables y la empatía. En cuanto a la cooperación condicionada y la empatía previa a la intervención, el coeficiente de correlación de Spearman es negativo 0.04 , lo cual indica que a mejor cooperación condicionada previa menor es la empatía previa.

Además, en la cooperación incondicionada y la empatía tras la intervención, dado que el p-valor es menor a .05 , rechazamos la hipótesis nula. Por consiguiente, existe suficiente evidencia estadística para afir- mar que la percepción sobre la cooperación incondicionada post y la empatía post está relacionada significativamente.

Tabla 6

\begin{tabular}{|c|c|c|c|c|}
\hline \multirow{2}{*}{ Antes } & \multirow{2}{*}{$\begin{array}{c}\text { Cooperación } \\
\text { Condicionada } \\
\end{array}$} & \multirow{2}{*}{$\begin{array}{c}\text { Cooperación } \\
\text { Incondicionada }\end{array}$} & \multicolumn{2}{|c|}{ Cooperación Situacional del Cooperación } \\
\hline & & & Alumnado & Total \\
\hline $\begin{array}{l}\text { Correlación } \\
\text { de Spearman }\end{array}$ & -.04 & .44 & .39 & .32 \\
\hline Sig. (bilateral) & .68 & .00 & .00 & .00 \\
\hline $\mathrm{N}$ & 107 & 107 & 107 & 107 \\
\hline Después & $\begin{array}{l}\text { Cooperación } \\
\text { Condicionada }\end{array}$ & Cooperación & \multicolumn{2}{|c|}{ Cooperación Situacional del Cooperación } \\
\hline $\begin{array}{r}\text { Correlación } \\
\text { de Spearman }\end{array}$ & 10 & .57 & .55 & .50 \\
\hline Pig. (bilateral) & .28 & .00 & .00 & .00 \\
\hline $\mathrm{N}$ & 107 & 107 & 107 & 107 \\
\hline
\end{tabular}

A su vez, el coeficiente de correlación de Spearman $=.57$, lo cual indica una relación positiva de nivel moderado, es decir, a mejor cooperación incondicionada mejor es empatía tras la intervención. Apreciándose una relación más positiva en el post que en el pre (.44 frente a .57 del post). El p-valor de la cooperación situacional del alumnado y la cooperación total vuelve a ser menor de .05 (tabla 6). Por lo tanto, volvemos a rechazar la hipótesis nula, pudiendo afirmar estadísticamente que la empatía está relacionada con las dos variables anteriormente citadas. En el caso de la cooperación situacional del alumnado, la correlación de Spearman es .55 y en la cooperación total .50, habiendo una relación moderada entre dichas variables y la empatía. Además, esta relación es más positiva tras la intervención que antes de la misma.

En la cooperación condicionada y la empatía tras la intervención se obtiene que el coeficiente de correlación de Spearman es positivo, lo cual indica que a mejor cooperación condicionada mejor es la empatía total.

\section{Decisiones de acción para la próxima pues- ta en práctica}

Debido a la escasez de estudios acerca de las conductas prosociales íntimamente relacionadas con la inteligencia interpersonal y a través de la actividad física y el deporte escolar, se invita a realizar trabajos acerca de otras conductas prosociales como son el Respeto, las Relaciones Sociales y la Capacidad de Liderazgo.

Otra futura línea que se plantea es el trabajo de otras conductas prosociales que estén vinculadas con la inteligencia emocional (Inteligencia Intrapersonal e Inteligencia Interpersonal) y mediante los deportes alternativos.

Asimismo, se invita a intervenir aplicando más sesiones para posteriormente valorar los resultados obtenidos, a realizar esta experiencia didáctica en otro contexto socio-económico, y con todos los cursos de secun- 
daria.

Por último, sería también interesante realizar una comparativa entre las emociones obtenidas en el alumnado durante la práctica de deportes tradicionales y deportes alternativos.

\section{Conclusiones}

En primer lugar, y en virtud de los resultados obtenidos, y aunque no se puede generalizar porque la muestra de la intervención es pequeña, se puede afirmar que los deportes alternativos, como el datchball y el copbol, aplicados en las clases de EF pueden ayudar a mejorar el desarrollo de la inteligencia interpersonal; ya que la empatía y la cooperación aumenta tras la realización de esta experiencia didáctica.

Por último, cabe mencionar, aunque no es el foco del estudio, que los resultados obtenidos en cuanto a sexo vislumbran una idea de que la práctica de los deportes alternativos, al estar enfocados desde una perspectiva formativa, lúdica y novedosa puede ayudar a disminuir la influencia de los dos modelos tradicionales imperantes: el masculino y el femenino, con sus correspondientes rasgos estereotipados. Y, por tanto, contribuye a la vivencia de emociones positivas en ambos sexos y a una mejora de las relaciones de género positivas en el estudiantado.

\section{Referencias}

Aguilar, M., García-Fernández, C., \& Gil del Pino, C. (2021). Efectividad de un programa educativo en Educación Física para fomentar las habilidades socioafectivas y prevenir la violencia en educación primaria. Retos: nuevas tendencias en educación física, deporte y recreación, 41, 492-501. Recuperado de https: / / doi.org/10.47197/retos.v0i41.82683

Asociación Internacional de Datchball, (2018). Datchball. Zaragoza, España. Recuperado de https:// www.datchball.com

Balcazar, A. (2020). El campamento educativo como escenario para el fortalecimiento de habilidades sociales. Retos: nuevastendencias en educación física, deporte y recreación, 41, 143-152. Recuperado de https:// doi.org/10.47197/retos.v0i41.82262

Bar-On, T., \& Parker, J. (2018). Inventario de Inteligencia Emocional de BarOn: versión para jóvenes. Bermejo, R., Ferrándiz, C., Ferrando, M., Prieto, MD, Sáinz, M., Eds. Recuperado de http:// www.web.teaediciones.com/Ejemplos/
BarOn_extracto-web.pdf

Bendicho, J. (2014). Colpbol, página oficial.Valencia, España. Recuperado de http://www.colpbol.es

Bermudez, C. \& Saenz-López, P. (2019). Emociones en Educación Física: una revisión bibliográfica (20152017). Retos: nuevas tendencias en educación física, deporte y recreación, (36), 597-603. Recuperado de https://recyt.fecyt.es/index.php/retos/article/ view $/ 70447 / 43968$

Constantino, S., \& Navia, J. (2021). Análisis del nivel de inclusión y posibles barreras de las técnicas de desarrollo de la inteligencia emocional y en particular del mindfulness en el área de Educación física en la ESO. Retos: nuevas tendencias en educación física, deporte y recreación, 41, 562-572. Recuperado de https:// doi.org/10.47197/retos.v0i41.85489

Cubas-Martínez,V., Marco-Ahulló, A., Monfort-Torres, G., Villarrasa-Sapiña, I., Pardo-Ibañez, A., \& GarcĺaMasso, X. (2019). Perfiles de actividad física, obesidad, autoestima y relaciones sociales del alumnado de primaria: un estudio piloto con Self-Organizing Maps. Retos: nuevas tendencias en educación física, deporte y recreación, 36, 146-151. Recuperado de https:// doi.org/10.47197/retos.v36i36.67549

Eisenberg, N., Zhou, Q., Spinrad, T.L., Valiente, C., Fabes, R. \& Liew, J. (2005). Relations among positive parenting, children's effortful control, and externalizing problems: A three-wave longitudinal study. Child Development, 76(5), 1055-1071. Recuperado de https://doi.org/10.1111/j.14678624.2005.00897.x

Escamilla, P., Alguacil, M. \& González-Serrano, M. (2020). Variables predictoras de la motivación deportiva en estudiantes de secundaria federados y no federados Retos: Nuevas tendencias en educación física, deporte y recreación, (38), 58-65. Recuperado de https: / /doi.org/10.47197/retos.v38i38.73551

Extremera, N., \& Fernández-Berrocal, P. (2003). La inteligencia emocional en el contexto educativo: hallazgos científicos de sus efectos en el aula. Revista de educación, 332(12), 97-116. Recuperado de http:// www.educacionyfp.gob.es/dam/jcr:6b5bc679e550-47d9-804e-e86b8f4b4603/re3320611443pdf.pdf

Extremera, N. \& Fernández-Berrocal, P. (2004). El papel de la inteligencia emocional en el alumnado: evidencias empíricas. Revista electrónica de investigación educativa, 2(6), 1-18. Recuperado de http:// www.redalyc.org/pdf/155/15506205.pdf

Fernández-Berrocal, P. \& Extremera, N. (2002). La in- 
teligencia emocional como una habilidad esencial en la escuela. Revista Iberoamericana de educación, 29(1), 1-6. Recuperado de https://doi.org/10.35362/ rie2912869

Gardner, H. (1995). Inteligencias múltiples. (1 a ed.). Barcelona, España: Paidós.

George, D. \& Mallery, P. (1995). SPSS / PC+ step by step: A simple guide and reference (p.168). Belmont: Wadsworth Publishing Company. Recuperado de https: / /wps.ablongman.com/wps/media/objects/ 385/394732/george4answers.pdf

Goleman, D. (2015). El cerebro y la inteligencia emocional: nuevos descubrimientos. Barcelona, España: Ediciones B.

Hamadeh, M., \& Ramírez, E. (2017). Innovación educativa con deportes alternativos novedosos en estudiantes de Educación Secundaria Obligatoria. En Book of abstracts CIVINEDU 2017: 1st International Virtual Conference on Educational Research and Innovation (p.81). Eindhoven, Países Bajos: Adaya Press. Recuperado de http: / /www.adayapress.com/wp-content/ uploads/2017/10/CIVINEDU2017.pdf

Hernández, A., Martínez, I. \& Carrión, S. (2019). El Colpbol como un medio para incrementar la motivación en Educación Primaria. Retos: nuevas tendencias en educación física, deporte y recreación, (36), 348-353. Recuperado de https://doi.org/10.47197/ retos.v36i36.70396

Leo, F. M., Sánchez-Miguel, P. A., Sánchez-Oliva, D., Amado, D., \& García-Calvo, T. (2011). Incidencia de la cooperación, la cohesión y la eficacia colectiva en el rendimiento en equipos de fútbol. Revista Internacional de Ciencias del Deporte, 26(7), 341-354. Recuperado de https://doi.org/10.5232/ ricyde2011.02601

Lizandra, J., \& Peiró-Velert, C. (2019). Las relaciones sociales y su papel en la motivación hacia la práctica de actividad física en adolescentes: Un enfoque cua- litativo. Retos: nuevas tendencias en educación física, deporte y recreación, 37, 41-47. Recuperado de https: / / doi.org/10.47197/retos.v37i37.70374

Martorell, M. C., González, R., \& Calvo, A. J. (1998). Cuestionario de conducta prosocial. Valencia, España: Universidad de Valencia.

Mayer, J., \& Cobb, C. (2000). Educational policy on emotional intelligence: the case for ability scales. Educational psychology review, 12(2), 163-183. Recuperado de https: / / psycnet.apa.org/record/200100355-015

Molero, C., Saiz, E. \& Esteban, C. (1998). Revisión histórica del concepto de inteligencia: una aproximación a la inteligencia emocional. Revista latinoamericana de Psicología, 30(1), 11-30. Recuperado de h t t p s : / / w w w. red a l y c.org / articulo.oa?id $=80530101$

Rey, M. (2009). La cooperación en el aula. Revista Innovación y Experiencia Educativas, 4. Recuperado de https: / / archivos.csif.es / archivos / andalucia/ ensenanza/revistas/csicsif/revista/pdf / Numero_14/MARIA\%20DEL\%20MAR_REY_2.pdf

Roche, R. (2004). Desarrollo de la inteligencia emocional y social desde los valores y actitudes prosociales en la escuela (1a ed.). Buenos Aires, Argentina: Ciudad Nueva.

Ros, A., Moya-Faz, F. J., \& Garcés de los Fayos, E. J. (2013). Inteligencia emocional y deporte: situación actual del estado de la investigación. Cuadernos de psicología del deporte, 13(1), 105-112. Recuperado de https: / / revistas.um.es/cpd/article/view / 177241

Thayer, R. (2003). Calm energy: how people regulate mood with food and exercise. Oxford, Reino Unido: Oxford University Press.

Weisinger, H. (1999). Emotional intelligence at work: The untapped edge for success. Performance Improvement, 38(6), 39-42. Recuperado de https: / / doi.org/10.1002/pfi.4140380611

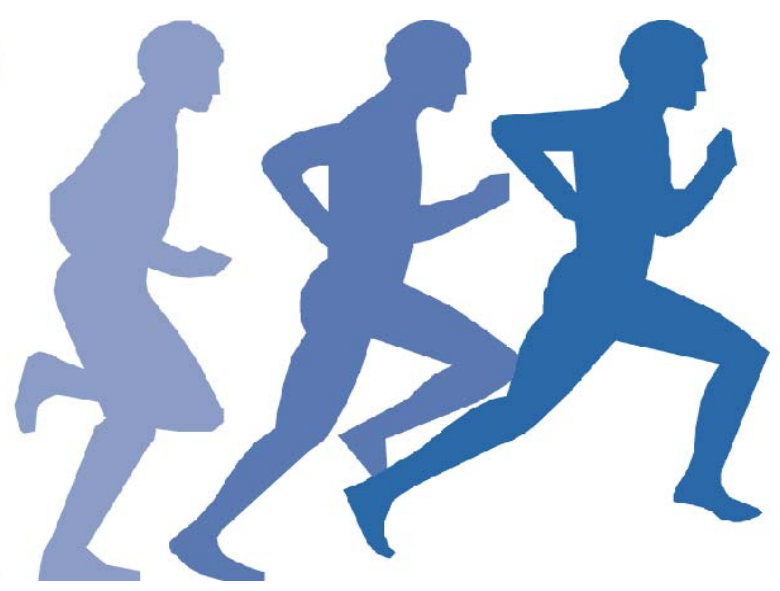

